\title{
Selection of mosquito life-histories: a hidden weapon against malaria?
}

Heather M Ferguson ${ }^{1 *}$, Nicolas Maire ${ }^{2,3}$, Willem Takken ${ }^{4}$, Issa N Lyimo ${ }^{5}$, Olivier Briët ${ }^{2,3}$, Steve W Lindsay ${ }^{6}$ and Thomas A Smith ${ }^{2,3}$

\begin{abstract}
Background: There has recently been a substantial decline in malaria incidence in much of Africa. While the decline can clearly be linked to increasing coverage of mosquito vector control interventions and effective drug treatment in most settings, the ubiquity of reduction raises the possibility that additional ecological and associated evolutionary changes may be reinforcing the effectiveness of current vector control strategies in previously unanticipated ways.

Presentation of hypothesis: Here it is hypothesized that the increasing coverage of insecticide-treated bed nets and other vector control methods may be driving selection for a shift in mosquito life history that reduces their ability to transmit malaria parasites. Specifically it is hypothesized that by substantially increasing the extrinsic rate of mortality experienced in vector populations, these interventions are creating a fitness incentive for mosquitoes to re-allocate their resources towards greater short-term reproduction at the expense of longer-term survival. As malaria transmission is fundamentally dependent on mosquito survival, a life history shift in this direction would greatly benefit control.
\end{abstract}

Testing the hypothesis: At present, direct evaluation of this hypothesis within natural vector populations presents several logistical and methodological challenges. In the meantime, many insights can be gained from research previously conducted on wild Drosophila populations. Long-term selection experiments on these organisms suggest that increasing extrinsic mortality by a magnitude similar to that anticipated from the up-scaling of vector control measures generated an increase in their intrinsic mortality rate. Although this increase was small, a change of similar magnitude in Anopheles vector populations would be predicted to reduce malaria transmission by $80 \%$.

Implications of hypothesis: The hypothesis presented here provides a reminder that evolutionary processes induced by interventions against disease vectors may not always act to neutralize intervention effectiveness. In the search for new intervention strategies, consideration should be given to both the potential disadvantages and advantages of evolutionary processes resulting from their implementation, and attempts made to exploit those with greatest potential to enhance control.

Keywords: Anopheles vectors, Life history evolution, Malaria, Insecticide-treated bed nets, Extrinsic mortality, Natural selection

\section{Background}

There has recently been a substantial decline in malaria incidence in Africa [1,2]. Some of the decline can be explained by the massive deployment of insecticide-treated bed nets (ITNs), the introduction of artemisinin

\footnotetext{
* Correspondence: Heather.Ferguson@glasgow.ac.uk

'Boyd Orr Centre for Population and Ecosystem Health, University of

Glasgow, Glasgow G12 8Q, UK

Full list of author information is available at the end of the article
}

combination therapy (ACT), or in some places, by the use of indoor residual spraying (IRS). But these intervention effects alone do not explain all the changes that have been seen. In some places, vector populations have fallen, despite the absence of organized vector control programmes [3], while in others there has been no decline at all [4].

Scaling-up of interventions creates the risk that parasites will develop resistance to drugs $[5,6]$, or that

\section{Biomed Central}


vectors will develop chemical $[7,8]$ or behavioural resistance [9-11]. However, not all selective pressures generated by interventions make malaria control more difficult. For instance, there is some evidence that parasites can be selected for renewed sensitivity to chloroquine [12]; albeit potentially on a short-term basis. Other pressures placed upon mosquito vectors by the rapid scaling-up of ITNs and IRS may have potential to drive selection for new behaviours or phenotypes that could reduce their capacity to transmit.

\section{Presentation of hypothesis}

The major African vectors Anopheles gambiae sensu latu and Anopheles funestus have adapted over many generations to feed on sleeping humans in mud-brick dwellings, and then rest indoors while digesting their blood meal. This behaviour is under threat from the massive scalingup of ITNs and IRS [2] which makes host-seeking and resting inside houses far more hazardous for mosquitoes. Concurrently, economic development is improving living conditions in many African countries, and increasing the prevalence of houses with corrugated-iron roofs, brick walls and screened windows that are more difficult for mosquitoes to enter and provide poor resting places for them [13]. These factors combine to increase the fitness costs associated with feeding on humans (anthropophagy), and have potential to generate selection for a shift towards feeding on non-human animals and/or biting and resting outdoors[14-16]. Such shifts may be especially likely if the fitness costs associated with adopting such novel phenotypes are minor [17].

An additional, previously unconsidered and potentially beneficial evolutionary consequence of control measures targeted at the main anthropophilic vectors of malaria in Africa is the selection for a shift in life history that reduces their ability to sustain parasite transmission. Like most organisms, mosquito vectors face trade-offs between investment in reproduction and survival [18], with malaria parasites being intimately dependent on the latter. This is because parasites, such as Plasmodium falciparum, require at least 12 days for development inside their vectors before they can infect a new host [19]. However, mosquito longevity in the wild is often low, with $<10 \%$ of An. gambiae sensu strictu females surviving long enough for parasites to complete their incubation [20]. Consequently any mosquito life-history shift in favour of short-term reproduction at the expense of longer-term survival would greatly reduce mosquito transmission potential. Evolutionary-based approaches to reduce malaria transmission by targeting mosquito survival have been recently proposed in the context of late-life acting insecticides [e.g. [21-23]]. This approach is thematically linked to the hypothesis presented here in its general aim of deploying vector control interventions that do not prompt a detrimental evolutionary response in mosquitoes that would hinder control (e.g. insecticide resistance). However these approaches differ in that the 'evolution-proof' approach requires that the control approach places little or no selection on mosquitoes, whereas the current hypothesis requires the generation of selection but in the direction of reducing intrinsic mosquito survival.

\section{Testing the hypothesis}

Evolutionary theory [24,25] and empirical studies demonstrate that increased exposure to sources of extrinsic mortality can generate selection for increased intrinsic mortality [26,27]. In brief, if external factors make the odds of long life minimal then organisms would be pushed to prioritize early reproduction at the expense of longevity. Laboratory studies have demonstrated that this phenomenon can occur in arthropods exposed to life-shortening pathogens [28]. By similarly increasing extrinsic mortality, the dissemination of insecticidal interventions could thus also place intense selection on malaria vectors for increased intrinsic mortality. Since malaria transmission is highly sensitive to the survival of the adult female mosquito, even a small increase in intrinsic mortality could have profound epidemiological benefits [29]. Indeed, this represents the theoretical rationale for the use of both IRS and ITNs as tools for reducing malaria transmission.

Unfortunately, testing this hypothesis is not easy. A comparison of the fecundity and intrinsic mortality of different wild-caught mosquito populations in relation to the extrinsic mortality rates would be logistically challenging. Recent progress in the establishment of semi-field systems for experimental study of mosquito ecology [e.g. [30]] may increase the feasibility of comparing intrinsic mortality in progeny of wild-caught mosquitoes. However, estimating the relative contribution of intra-specific genetic variation to geographical differences in mosquito mortality, given their ecological and taxonomic complexity, will remain a challenge. As the methodological approaches for study of these phenomena in wild vector populations develops, some a priori hypotheses can be generated from research previously conducted on wild Drosophila populations [26]; dipterans often used as model organisms for Anopheles genetics and physiology.

In a series of long-term selection experiments conducted in an insectary, Stearns and colleagues [26] exposed a variety of strains of Drosophila to different extrinsic mortality regimes over a period of five years (50-90 generations). Selection under high extrinsic mortality resulted in both an increase in fecundity, and a small increase in (intrinsic) mortality when assayed in the absence of selection (Figure 1)[26]. While for the 


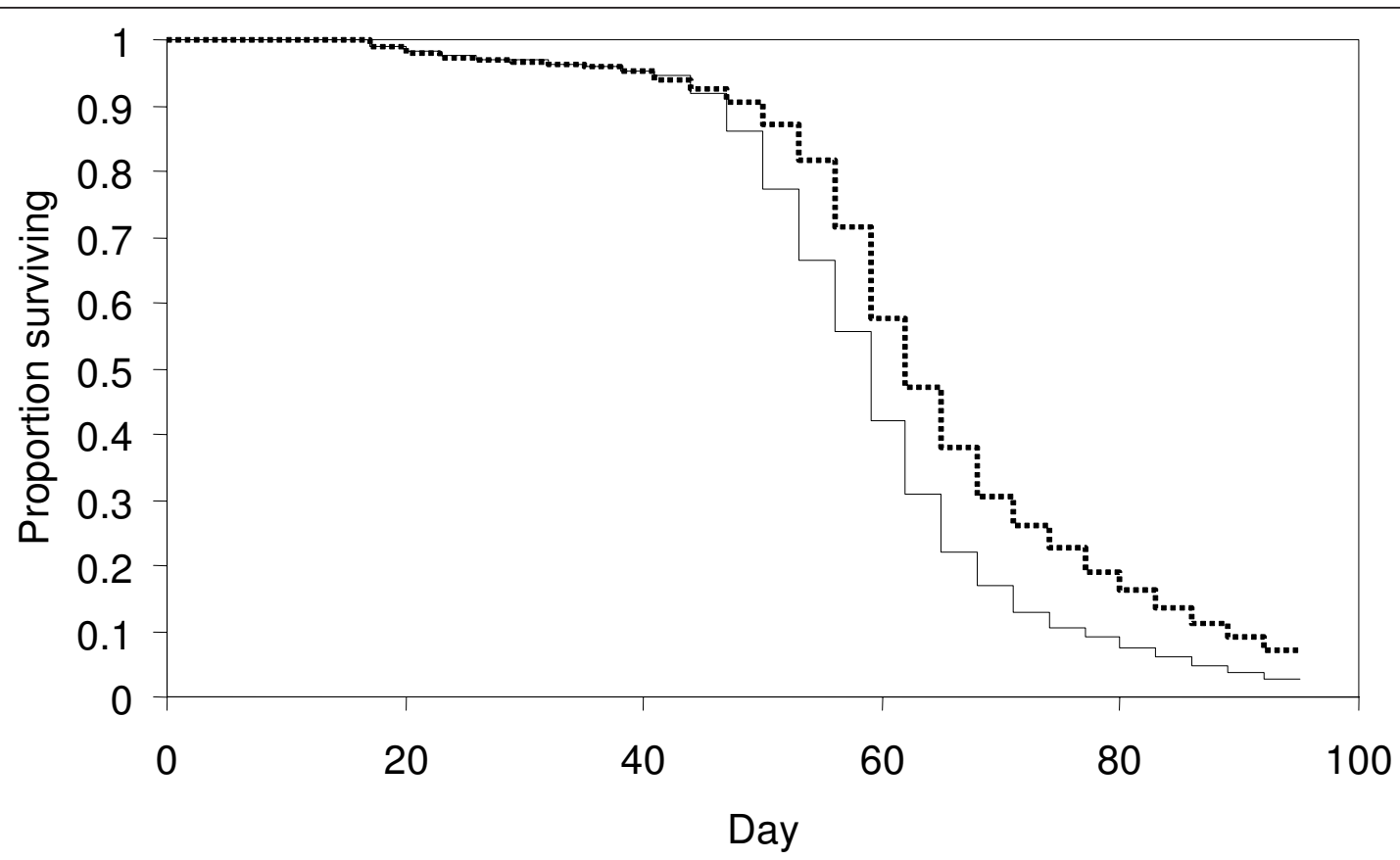

Figure 1 Kaplan-Meier curves comparing intrinsic mortality of Drosophila populations after selection. Dashed line: low extrinsic mortality selection regime; continuous line: high extrinsic mortality selection regime. Data replotted from [26].

high intrinsic mortality lines the median survival time (58.4 days) was only $7.7 \%$ lower than that of the low intrinsic mortality lines (63.3 days), this difference in relative survival would correspond to an approximately $80 \%$ reduction in transmission in a typical endemic setting (Figure 2) given the non-linear relationship between mosquito survival and the vectorial capacity described originally by Garrett-Jones [31], who showed that within the Ross-MacDonald model a reduction in daily survival probability of the vector from $p_{1}$ to $p_{2}$ results in a reduction in vectorial capacity from $C_{1}$ to $C_{2}$ as:

$$
\frac{C_{2}}{C_{1}}=\left(\frac{p_{2}}{p_{1}}\right)^{n} \frac{\log _{e}\left(p_{1}\right)}{\log _{e}\left(p_{2}\right)}
$$

where $n$ is the number of days required for the parasite to complete its extrinsic incubation period [31]. On this basis it appears possible that increased mosquito extrinsic mortality induced by the high coverage of long lasting insecticide treated nets (LLINs) that has now been achieved in many parts of sub-Saharan Africa could select for increased intrinsic mortality with substantial impact on malaria transmission over and above the immediate protection that LLINs provide to communities.

\section{Implications of hypothesis}

The theoretical possibility that such selection could occur does not demonstrate that it is playing a role in recent reductions in malaria transmission in Africa. Amongst other considerations, the magnitude of the hypothesized survival effect depends on all other epidemiologically-relevant aspects of vector ecology remaining the same (e.g. the vector-human ratio, blood feeding rate on humans, duration of sporogony). Any correlated change in these parameters prompted by intervention use could magnify or diminish the transmission effects proposed here. For example, the enhanced investment in short-term reproduction hypothesized here could be manifested as an increase in the number of eggs laid in one clutch. This could intensify competition during larval development, which through its connection with mosquito population growth [32] and adult survival [33] might prompt even greater transmission reduction. Proof of the existence and epidemiological importance of such a phenomenon will require a multi-pronged approach including at least: (1) confirmation of the heritability of intrinsic mortality and reproductive schedule in wild vector populations [34]; (2) evidence of standing genetic variation in these traits; (3) demonstration that the fitness costs imposed by these interventions are of sufficient magnitude to alter the fecundity-longevity trade-off; and (4) development of better tools for tracking the age and fecundity of mosquitoes in natural populations, and their response to increases in vector control coverage. Furthermore, as some empirical studies have shown that extrinsic mortality 


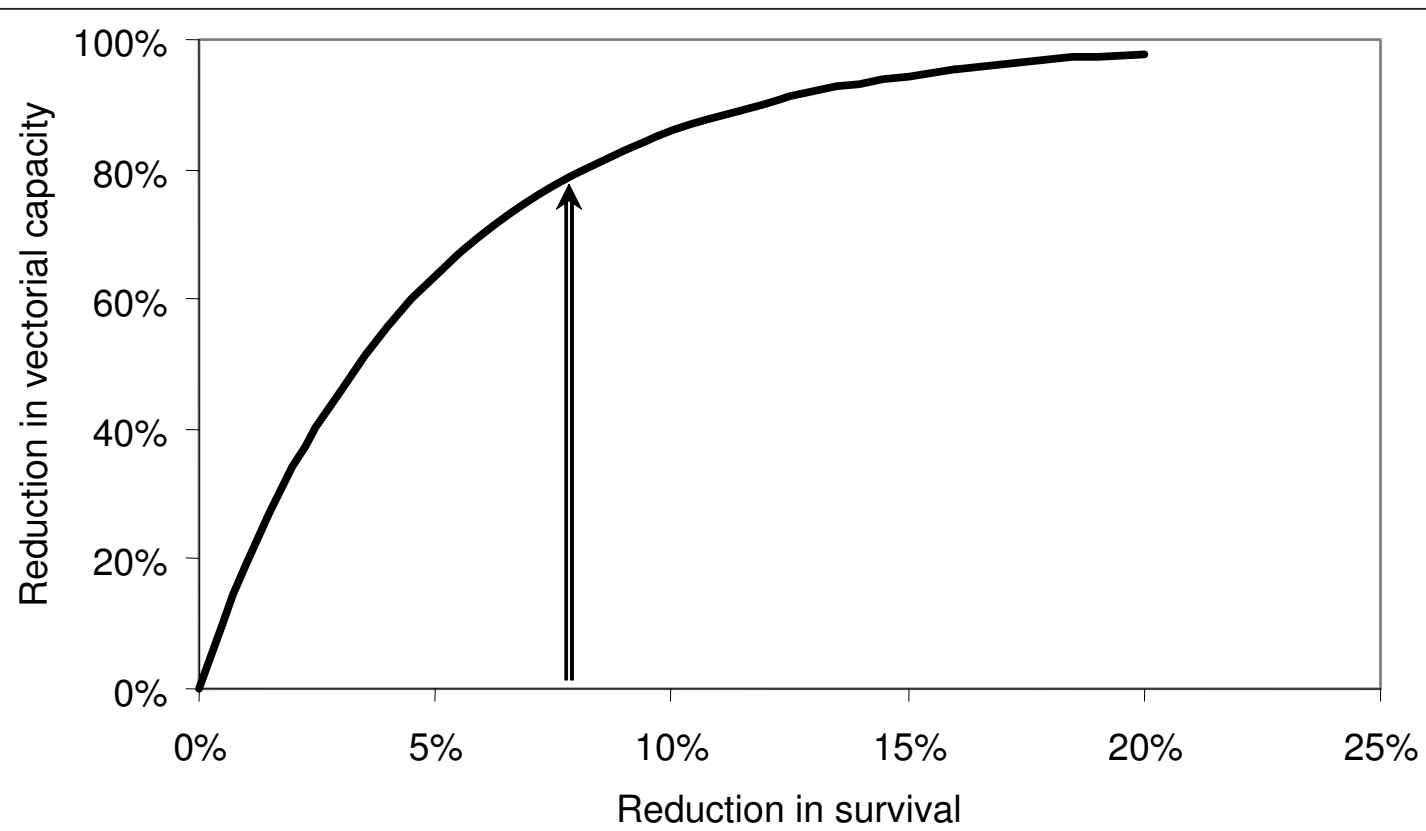

Figure 2 Predicted relationship between reductions in adult malaria vector survival and their transmission potential. The reduction in vectorial capacity was calculated using Garrett-Jones' original formula for the vectorial capacity, assuming a daily survival of $90 \%$ in the original vector population, and a 12-day duration of malaria sporogony. The vertical arrow corresponds to a $7.7 \%$ reduction in survival.

pressure can select for a net increase in lifetime reproductive success in the absence of pressure [35], caution would be required to ensure that any epidemiological advantages arising from increased vector mortality would not be undermined by an upsurge in the size of their populations.

The possibility of the phenomena described here has important consequences. It provides a reminder that the evolutionary processes induced by interventions against disease vectors may not always act to neutralize intervention effectiveness. Secondly, it argues that large-scale distribution of highly effective interventions could have unpredictable effects. The selective pressures on life history traits depend on local ecology, for example, mosquitoes with high innate fecundity may have the greatest advantage in areas of abundant rainfall where larval habitat is not limiting, whereas in areas with long dry seasons selection may favour mosquito longevity over short-term reproduction [36]. Mosquito evolutionary responses to insecticidal interventions against malaria (e. g. insecticide resistance) have been correlated with a reduction in their ability to transmit other pathogens [37]; suggesting that the selection imposed by these measures could have potentially numerous unanticipated effects on disease risk. The complexity of the parasitevector-human-environment system will continue to present challenges for prediction. In the search for new control strategies, wide consideration should be given to both the potential epidemiological disadvantages and advantages of evolutionary processes resulting from their implementation.

\section{Acknowledgements}

HMF is funded by a BBSRC David Phillips Fellowship. INL was supported by a Mshinda Fellowship, WT by travel support by the Uyttenboogaart-Eliasen Foundation, The Netherlands, and SWL by the Research and Policy for Infectious Disease Dynamics (RAPIDD) Program of the Science and Technology Directory, Department of Homeland Security, and Fogarty International Center, National Institutes of Health. TS, OJTB, and NM are partly supported by the Bill and Melinda Gates Foundation.

\section{Author details}

'Boyd Orr Centre for Population and Ecosystem Health, University of Glasgow, Glasgow G12 8Q, UK. '2Department of Epidemiology and Public Health, Swiss Tropical and Public Health Institute, PO Box, CH-4002, Basel, Switzerland. ${ }^{3}$ University of Basel, PO Box, CH-4003, Basel, Switzerland. ${ }^{4}$ Laboratory of Entomology, Wageningen University, 6700 EH Wageningen, The Netherlands. ${ }^{5}$ Biomedical and Environmental Thematic Group, Ifakara Health Institute, Ifakara, PO Box 53, Tanzania. ${ }^{6}$ School of Biological and Biomedical Sciences, Durham University, Durham DH1 3LE, UK.

\section{Authors' contributions}

All authors contributed to the development of this hypothesis, and provided comments on the manuscript. HMF and TAS drafted the manuscript. All authors read and approved the final manuscript.

\section{Competing interests}

The authors declare that they have no competing interests.

Received: 19 January 2012 Accepted: 3 April 2012

Published: 3 April 2012

\section{References}

1. O'Meara WP, Mangeni JN, Steketee R, Greenwood B: Changes in the burden of malaria in sub-Saharan Africa. Lancet Infect Dis 2010, 10:545-555. 
2. World Malaria Report 2010. World Health Organization. Geneva; 2010.

3. Meyrowitsch DW, Pedersen EM, Alifrangis M, Scheike TH, Malecela MN, Magesa SM, Derua YA, Rwegoshora RT, Michael E, Simonsen PE: Is the current decline in malaria burden in sub-Saharan Africa due to a decrease in vector population? Malar J 2011, 10.

4. Okiro EA, Bitira D, Mbabazi G, Mpimbaza A, Alegana VA, Talisuna AO, Snow RW: Increasing malaria hospital admissions in Uganda between 1999 and 2009. BMC Med 2011, 9.

5. Mackinnon MJ, Marsh K: The selection landscape of malaria parasites. Science 2010, 328:866-871.

6. O'Brien C, Henrich PP, Passi N, Fidock DA: Recent clinical and molecular insights into emerging artemisinin resistance in Plasmodium falciparum. Curr Op Infect Dis 2011, 24:570-577.

7. Ranson H, N'Guessan R, Lines J, Moiroux N, Nkuni Z, Corbel V: Pyrethroid resistance in African anopheline mosquitoes: what are the implications for malaria control? Trends Parasitol 2011, 27:91-98.

8. Flaxman AD, Fullman N, Otten MW, Menon M, Cibulskis RE, Ng M, Murray CJ, Lim SS: Rapid scaling up of insecticide-treated bed net coverage in Africa and its relationship with development assistance for health: a systematic synthesis of supply, distribution, and household survey data. PLoS Med 2010, 7.

9. Garrett-Jones C, Boreham P, Pant CP: Feeding habits of anophelines (Diptera: Culicidae) in 1971-1978, with reference to the human blood index: a review. Bull Entomol Res 1980, 70:165-185.

10. Sampath TRR, Yadav RS, Sharma VP, Adak T: Evaluation of lambdacyhalothrin-impregnated bednets in a malaria endemic area of India. Part 2. Impact on malaria vectors. J Am Mosa Con Assoc 1998, 14:437-443.

11. Sharp BL, Lesueur D: Behavioral variation of Anopheles arabiensis (Diptera, Culicidae) populations in Natal, South Africa. Bull Entomol Res 1991, 81:107-110.

12. Wangai LN, Kimani FT, Omar SA, Karanja SM, Nderu DW, Magoma G, Mutua D: Chloroquine resistance status a decade after: Re-emergence of sensitive Plasmodium falciparum strains in malaria endemic and epidemic areas in Kenya. J Protozool Res 2011, 21:20-29.

13. Lindsay SW, Emerson PM, Charlwood JD: Reducing malaria by mosquitoproofing houses. Trends Parasitol 2002, 18:510-514.

14. Hii JLK, Smith T, Vounatsou P, Alexander N, Mai A, Ibam E, Alpers MP: Area effects of bednet use in a malaria-endemic area in Papua New Guinea. Trans R Soc Trop Med Hyg 2001, 95:7-13.

15. Bogh C, Pedersen EM, Mukoko DA, Ouma JH: Permethrin-impregnated bednet effects on resting and feeding behaviour of lymphatic filariasis vector mosquitoes in Kenya. Med Vet Entomol 1998, 12:52-59.

16. Reddy MR, Overgaard HJ, Abaga S, Reddy VP, Caccone A, Kiszewski AE, Slotman MA: Outdoor host seeking behaviour of Anopheles gambiae mosquitoes following initiation of malaria vector control on Bioko Island, Equatorial Guinea. Malar J 2011, 10.

17. Lyimo IN, Keegan SP, Ranford-Cartwright LC, Ferguson HM: The impact of uniform and mixed species blood meals on the fitness of the mosquito vector Anopheles gambiae s.s: does a specialist pay for diversifying its host species diet? J Evol Biol 2012, 25:452-460.

18. Stearns SC: The Evolution of Life Histories New York: Oxford University Press; 1995.

19. Beier JC: Malaria parasite development in mosquitoes. Ann Rev Entomol 1998, 43:519-543.

20. Gillies MT, Wilkes TJ: A Study of Age-Composition of Populations of Anopheles gambiae Giles and $A$ funestus Giles in North-Eastern Tanzania. Bull Entomol Res 1965, 56:237-263.

21. Koella JC, Lynch PA, Thomas MB, Read AF: Towards evolution-proof malaria control with insecticides. Evol Appl 2009, 2:469-480.

22. Lorenz LM, Koella JC: The microsporidian parasite Vavraia culicis as a potential late life-acting control agent of malaria. Evol Appl 2011, 4:783-790

23. Glunt KD, Thomas MB, Read AF: The effects of age, exposure history and malaria infection on the susceptibility of Anopheles mosquitoes to low concentrations of pyrethroid. PLOS ONE 2011, 6:e24968.

24. Medawar PB: An unsolved problem of Biology London: H.K. Lewis; 1952

25. Williams GC: Pleioptropy, natural selection and the evolution of senescence. Evolution 1957, 11:398-411.
26. Stearns SC, Ackermann M, Doebeli M, Kaiser M: Experimental evolution of aging, growth, and reproduction in fruitflies. Proc Natl Acad Sci USA 2000, 97:3309-3313.

27. Gasser M, Kaiser M, Berrigan D, Stearns SC: Life-history correlates of evolution under high and low adult mortality. Evolution 2000, 54:1260-1272.

28. Agnew P, Koella JC, Michalakis Y: Host life history responses to parasitism. Microb Infect 2000, 2:891-896.

29. Macdonald G: The epidemiological basis of malaria control. Bull W H O 1956, 15:613-626.

30. Ng'habi KR, Mwasheshi D, Knols BG, Ferguson HM: Establishment of a selfpropagating population of the African malaria vector Anopheles arabiensis under semi-field conditions. Malar J 2010, 9:356.

31. Garrett-Jones C: The human blood index of malaria vectors in relation to epidemiological assessment. Bull W H O 1964, 30:241-261.

32. White MT, Griffin JT, Churcher TS, Ferguson NM, Basanez M-G, Ghani AC: Modelling the impact of vector control interventions on Anopheles gambiae population dynamics. Parasit Vect 2011, 4.

33. Russell TL, Lwetoijera DW, Knols BG, Takken W, Killeen GF, Ferguson HM: Linking individual phenotype to density-dependent population growth: the influence of body size on the population dynamics of malaria vectors. Proc $R$ Soc Biol Sci 2011, 278:3142-3151.

34. Lehmann T, Dalton R, Kim EH, Dahl E, Diabate A, Dabire R, Dujardin JP: Genetic contribution to variation in larval development time, adult size and longevity of starved adults of Anopheles gambiae. Infect, Gene Evoln 2006, 6:410-416.

35. Zwaan B, Bijlsma R, Hoekstra RE: Direct selection on life-span in Drosophila melanogaster. Evolution 1995, 49:649-659.

36. Omer SM, Cloudsle JL: Survival of female Anopheles gambiae Giles through a 9 month dry season in Sudan. Bull W H O 1970, 42:319-330.

37. McCarroll L, Hemingway J: Can insecticide resistance status affect parasite transmission in mosquitoes? Insect Biochem Molec Biol 2002, 32:1345-1351.

doi:10.1186/1475-2875-11-106

Cite this article as: Ferguson et al: Selection of mosquito life-histories: a hidden weapon against malaria? Malaria Journal 2012 11:106.

\section{Submit your next manuscript to BioMed Central and take full advantage of:}

- Convenient online submission

- Thorough peer review

- No space constraints or color figure charges

- Immediate publication on acceptance

- Inclusion in PubMed, CAS, Scopus and Google Scholar

- Research which is freely available for redistribution

Submit your manuscript at www.biomedcentral.com/submit
Ciomed Central 